\title{
A patient with an hourglass shaped fractured coronary stent
}

\author{
Andreas Y. Andreou ${ }^{1,2}$, Angelos Tyrlis ${ }^{3}$ \\ ${ }^{1}$ Department of Cardiology, Limassol General Hospital, Limassol, Cyprus \\ ${ }^{2}$ University of Nicosia Medical School, Nicosia, Cyprus \\ ${ }^{3}$ St Bartholomew's Hospital, Barts Health NHS Trust, London, UK
}

A 53-year-old female patient with a history of stent angioplasties in 2014 and coronary artery bypass graft surgery (CABG) to the left anterior descending and obtuse marginal arteries in 2016 underwent coronary angiography in July 2019 due to unstable angina. Both grafts were patent whereas the proximal right coronary artery (RCA) contained an intermediate in-stent stenosis which corresponded to an area of stent deformation with an inward displacement of the struts on both sides of the stent without discontinuity in the stent body (Figures $1 \mathrm{~A}, \mathrm{~B}$ ). The stenosis was associated with an instantaneous wave-free ratio of 1.0. Intravascular ultrasound (IVUS) examination (Figures 1,2 ) revealed a grossly distorted stent without strut malapposition or neointimal buildup (Figures $1 \mathrm{C}$ and 2 panels 2, 6), which harboured an area with ulcerated atheroma and sparse stent struts indicating stent fracture (SF) (Figures $1 \mathrm{D}$ and 2 panels 3-5). Proximal RCA angulation and hinge motion were observed in February 2014 before the successful implantation of a $3.5 \mathrm{~mm} \times 28 \mathrm{~mm}$ biolimus A9-eluting BioMatrix Flex (Biosensor, Morges, Switzerland) stent in order to treat a catheter-induced dissection (Figure 3). Coronary angiography performed before CABG in March 2016 showed a structurally intact proximal RCA stent (Figure 4). Consequently, chronic stent recoil (SR) secondary to loss of radial strength of the stent due to late ( $>1$ year) SF attributed to mechanical fatigue was diagnosed. Angioplasty with a $3.75 \mathrm{~mm}$ non-compliant balloon and then a $3.75 \mathrm{~mm}$ paclitaxel-coated balloon was undertaken successfully (Figure 5). The patient was discharged on dual antiplatelet therapy to be taken for at least 12 months. Follow-up angiography at 6 months showed a patent stent without recurrent SR (Figure 6).

Coronary SF constitutes an underdiagnosed cause of stent failure that has predominantly been associated with in-stent restenosis but also stent thrombosis [1, 2]. Most adverse clinical events have been associated with complete SF with a gap in the stent body; yet, reported cases of restenosis at the site of partial SF with underlying heavy calcification suggested that acquired underexpansion or distortion of the fractured stent may play a role in occurrence of adverse outcomes. The diagnosis of SF is usually made by conventional angiography when a complete (circumferential) or partial gap is created between the stent fragments, and the success of diagnosis depends on the gap length and stent radiopacity and on whether the acquisition was made from the plane perpendicular to the gap $[1,3]$. Yet, high resolution cine-angiography (e.g. StentBoost Subtract, Philips Healthcare, Best, the Netherlands), when available, facilitates SF identification by enhancing stent strut visualization [4]. Our patient had many of the factors predisposing to SF such as location of the lesion in the RCA, arterial angulation $\left(>45^{\circ}\right)$ and hinge motion at the site of the lesion and implantation of a long, stainless steel stent with limited conformability [1]. Furthermore, our patient showed chronic SR, which is a rarely observed late-acquired stent axial deformation causing $>20 \%$ decrease in the stent area or diameter; it has been shown in nearly $30 \%$ of SF lesions [5]. Therefore, we had a high index of suspicion for an angiographically silent SF and this enabled its diagnosis by means of IVUS. The SF was partial and co-localized with ulcerated atheroma, to the creation of which possibly contributed the abnormal blood flow and wall shear stress patterns owing to stent distortion. In the absence of ischaemia-producing restenosis at the SF site or a complete SF we refrained from repeat stenting that could probably aggravate the already present unfavourable loading conditions of the stent with resultant

\section{Corresponding author:}

Andreas Y. Andreou MD, FESC, FACC, FSCAI, Department of Cardiology, Limassol General Hospital, Limassol, Cyprus,

e-mail: y.andreas@yahoo.com

Received: 30.07.2020, accepted: 19.10.2020. 

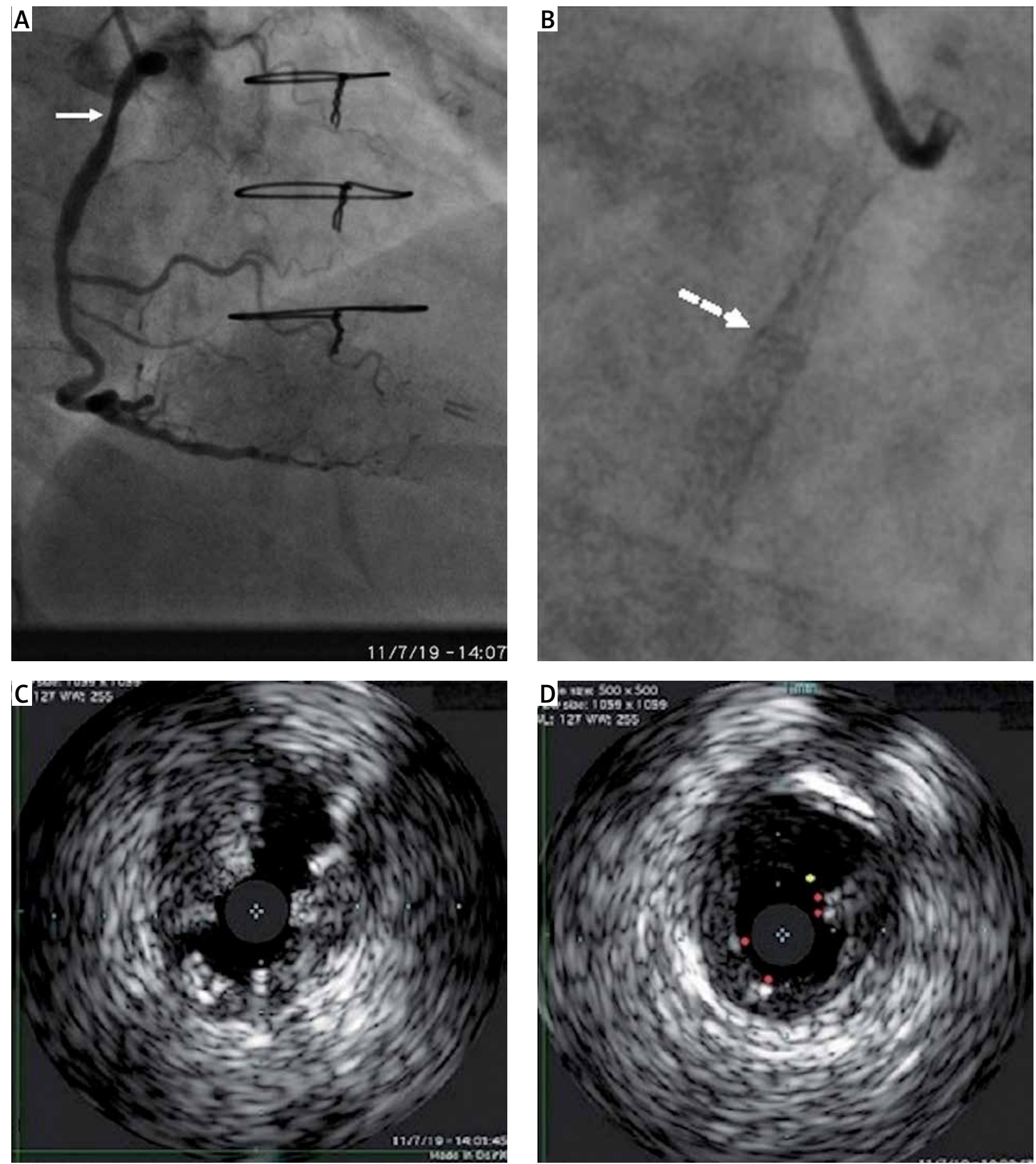

Figure 1. A - Angiogram (right anterior oblique (RAO) $30^{\circ}$ projection) depicting an intermediate in-stent stenosis (arrow) in the proximal right coronary artery (RCA) corresponding to stent recoil (SR). B - Contrast-free RCA angiogram (RAO $30^{\circ}$ projection) showing the area of SR that rendered the stent resembling an hourglass without any signs of stent fracture (SF); stent overlap (dotted arrow). C - Cross-sectional intravascular ultrasound (IVUS) image showing gross SR. D - Cross-sectional IVUS image showing sparse stent struts (red dots) indicating SF and eccentric soft (echolucent) atheroma with a recess beginning at the luminal-intimal border (yellow dot) suggesting plaque ulceration 


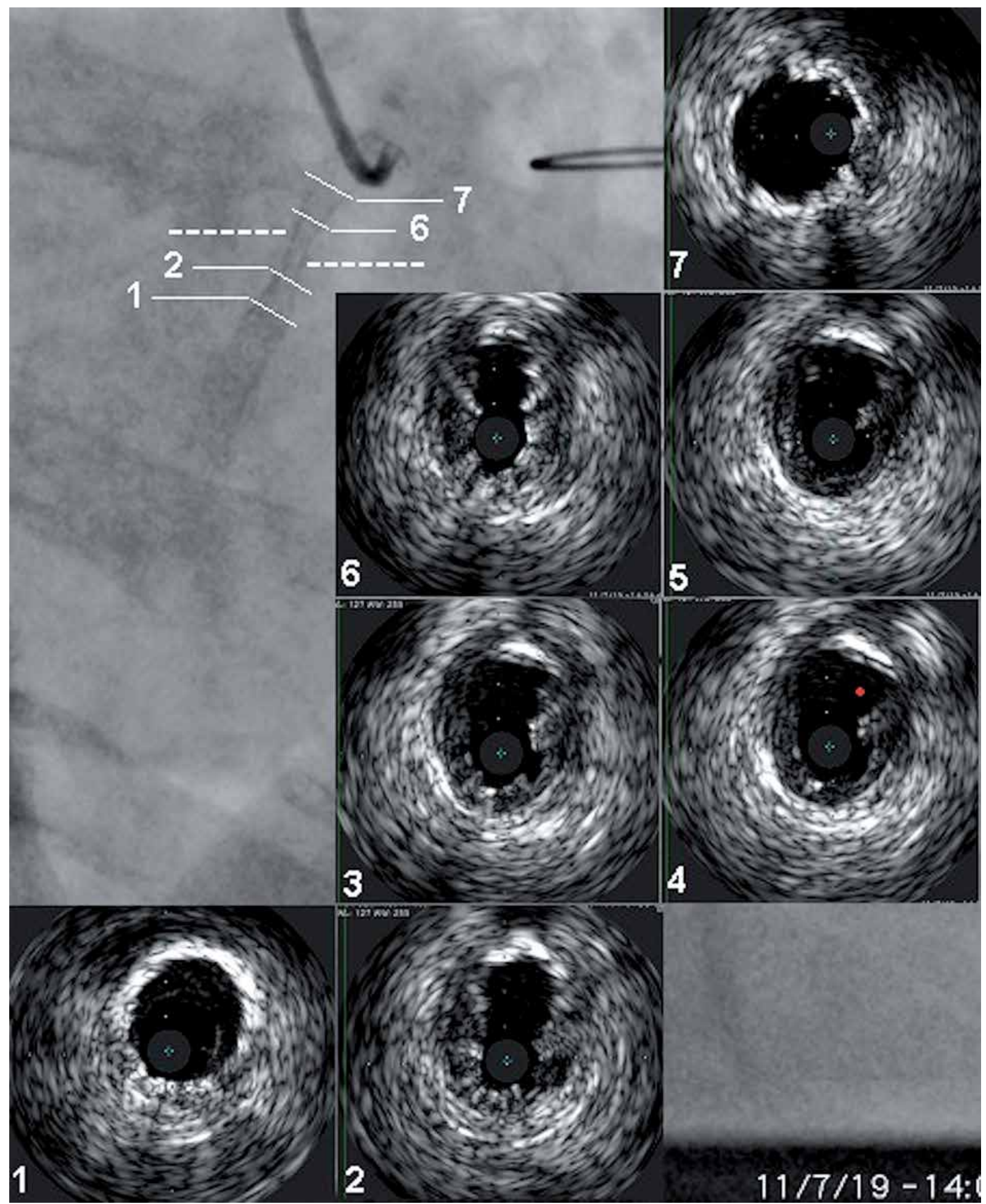

Figure 2. Intravascular ultrasound cross-sectional images (panels 1-7) of the proximal right coronary artery stent (distal to proximal). Note the symmetrically expanded distal (panel 1) and proximal (panel 7) parts of the stent bordering the part that exhibited recoil (panels 2 and 6). The site of stent fracture where sparse stent struts were recognized (panels 3 -5 between the dotted lines) together with an ulcerated atheromatous plaque best seen in panel 4 (red dot) is shown 

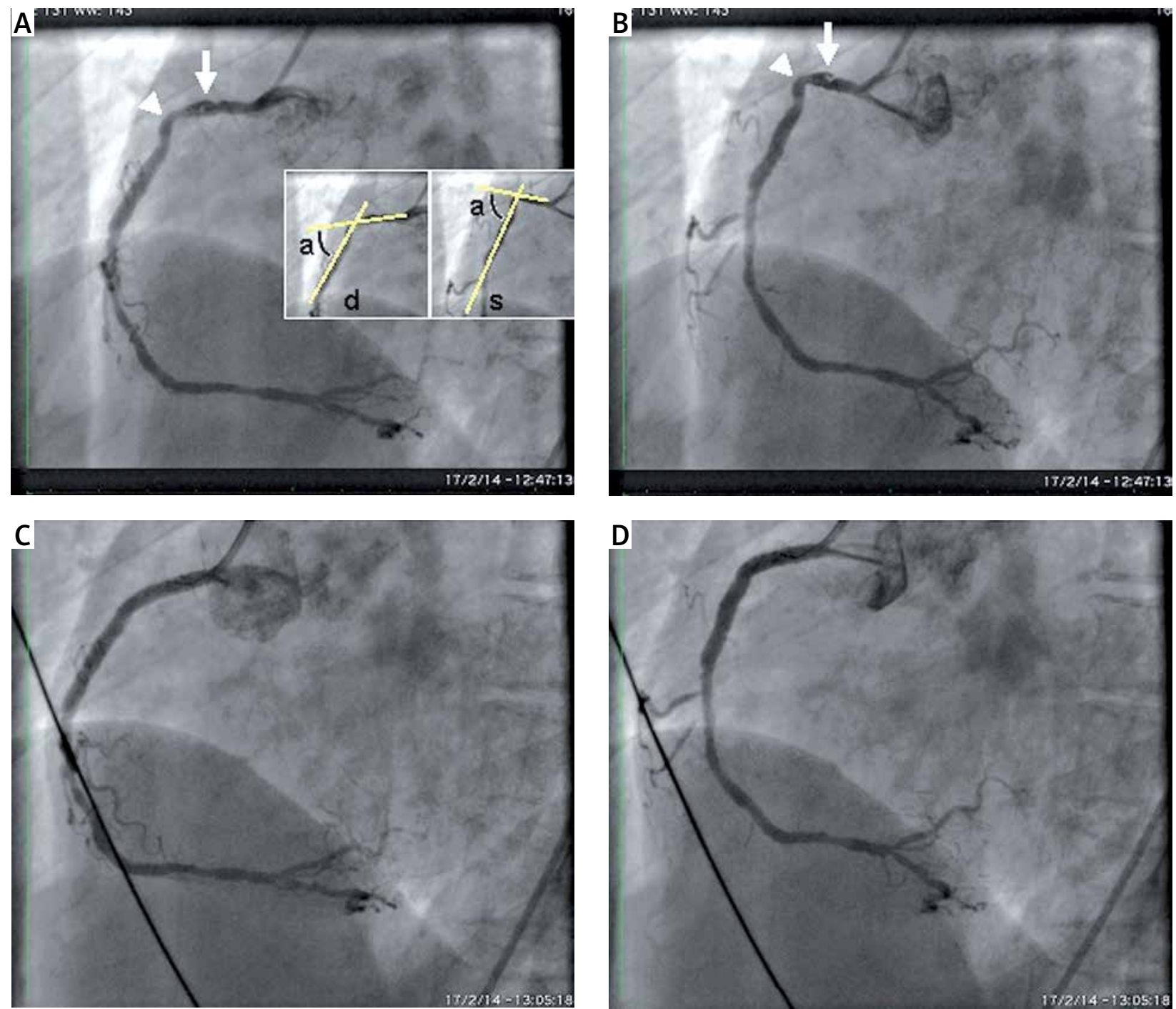

Figure 3. Selective right coronary artery angiograms in the left anterior oblique $45^{\circ}$ projection taken in February 2014. A, B - Diastolic and systolic frames respectively depicting the catheter-induced dissection in the proximal segment (arrow) that necessitated treatment by stent implantation. Note the angulated proximal course of the artery (arrowhead) just beyond the site of dissection. The marked displacement of the proximal segment during the cardiac cycle as well as the hinge motion (embedded panel; note the difference in angle a between diastole [d] and systole [s] which must be at least $16^{\circ}$ in hinge motion lesions) at the site of proximal arterial angulation is also evident. C, D - Diastolic and systolic frames respectively after stent implantation. Vessel straightening after stenting is evident in the diastolic frame

recurrent SF. Angioplasty with properly sized balloons including a drug-coated balloon was performed instead, with a favourable outcome.

This case highlights that stents prone to fracture such as those constructed from stainless steel should not be implanted across tortuous and hinge motion lesions that entail a high risk of fracture. Since fatigue SF is more likely to be discovered at progressively later timepoints after the index stent angioplasty, continued surveillance and maintenance of a high index of suspicion for SF are required. Also, the threshold for use of intravascular imaging modalities such as IVUS in suspicious cases should be low; this way, one can diagnose SF more accurately compared with conventional angiography but also, as highlighted herein, gain insights into the pathophysiology of SF-related adverse clinical events.

\section{Conflict of interest}

The authors declare no conflict of interest. 

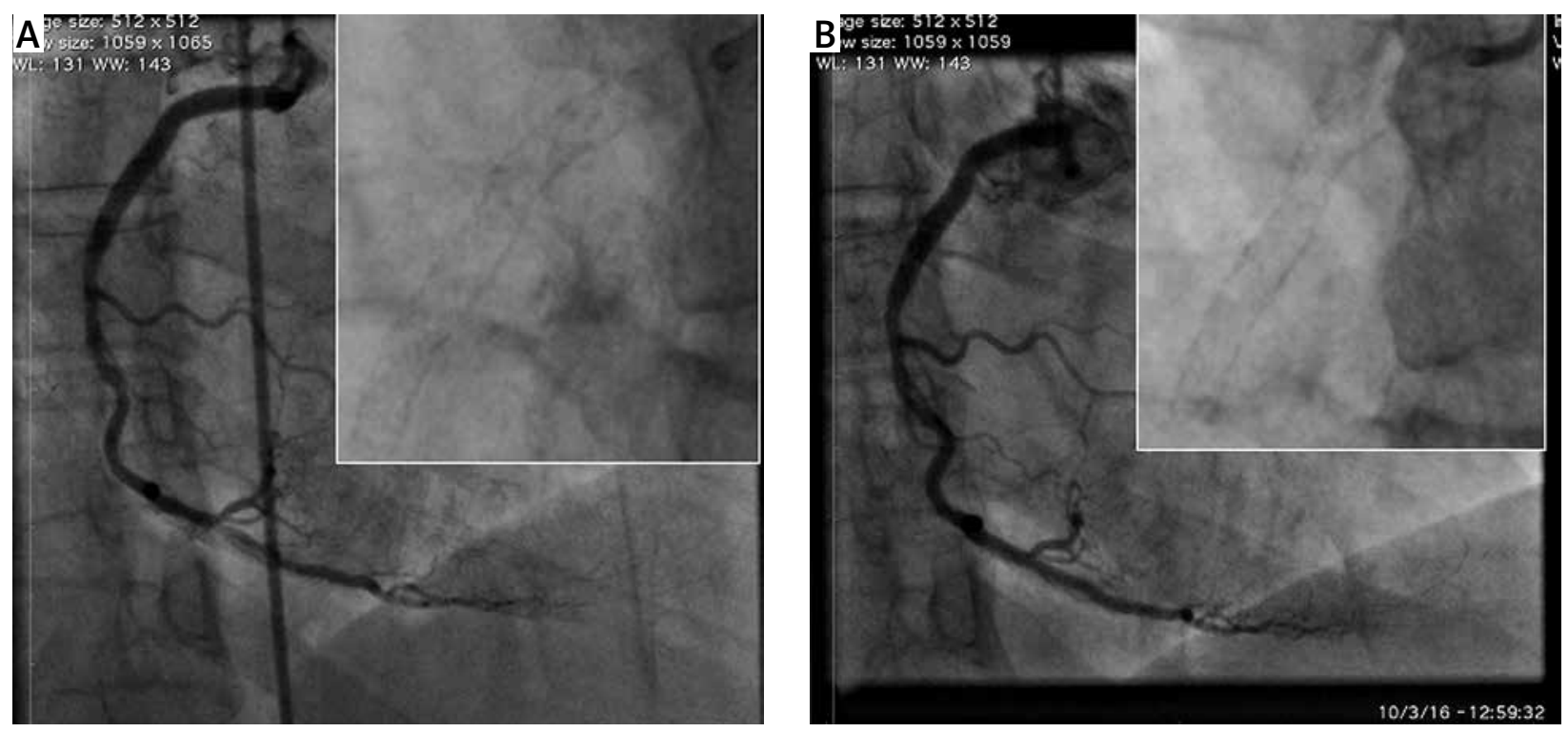

Figure 4. A, B - Right coronary artery angiograms in the right anterior oblique $30^{\circ}$ projection and contrast-free images in the posteroanterior cranial 20 projection (embedded panels) obtained in 20141.5 months after index stent implantation and in 2016 before coronary artery bypass surgery respectively showing a patent and structurally preserved proximal stent
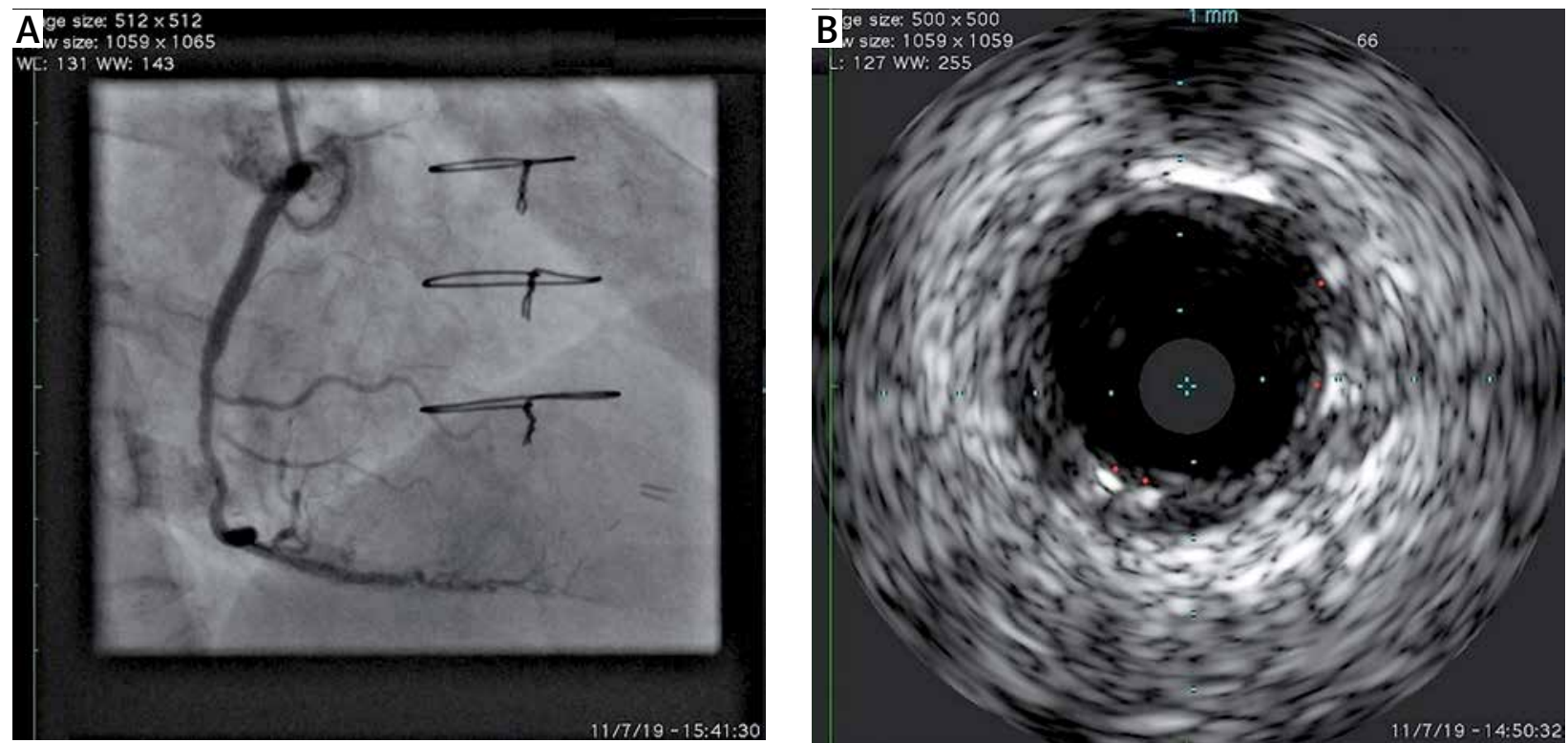

Figure 5. A - Angiogram of the right coronary artery in the right anterior oblique $30^{\circ}$ projection depicting the good result of angioplasty to the fractured and recoiled proximal stent. B - Cross-sectional intravascular ultrasound image corresponding to the site of stent fracture showing restoration of a circular and wide arterial lumen. Note the sparse stent struts (red dots) and stent strut crowding at 12 o'clock 

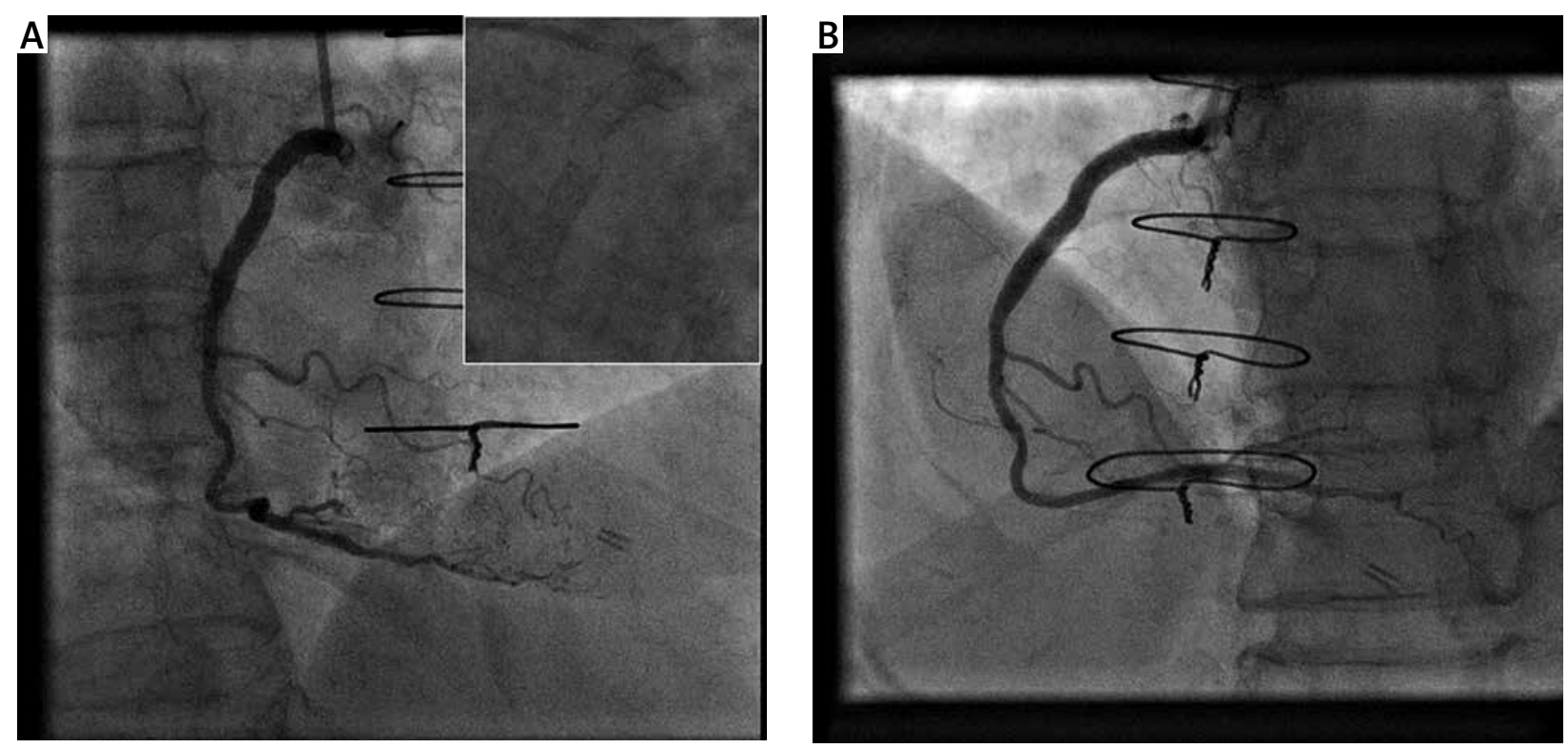

Figure 6. Six-month follow-up angiograms of the right coronary artery depicting a widely patent stent. A - Right anterior oblique $30^{\circ}$ projection. Contrast-free angiographic image showing no signs of recurrent stent recoil (embedded panel). $\mathbf{B}$ - Posteroanterior cranial $20^{\circ}$ projection

\section{References}

1. Omar A, Pendyala LK, Ormiston JA, Waksman R. Review: stent fracture in the drug-eluting stent era. Cardiovasc Revasc Med 2016; 17: 404-11.

2. Nakazawa G, Finn AV, Vorpahl M, et al. Incidence and predictors of drug-eluting stent fracture in human coronary artery a pathologic analysis. J Am Coll Cardiol 2009; 54: 1924-31.

3. Hecht HS, Polena S, Jelnin V, et al. Stent gap by 64-detector computed tomographic angiography relationship to in-stent restenosis, fracture, and overlap failure. J Am Coll Cardiol 2009; 54: 1949-59.

4. Biscaglia S, Tumscitz C, Tebaldi M, et al. Enhanced stent visualization systems during $\mathrm{PCl}$ : A case series and review of literature. J Cardiol Cases 2015; 12: 1-5.

5. Ohya M, Kadota K, Kubo S, et al. Incidence, predictive factors, and clinical impact of stent recoil in stent fracture lesion after drug-eluting stent implantation. Int J Cardiol 2016; 214 123-9. 